\title{
Enrique Bustamante: un maestro en comunicación
}

\author{
Guillermo Mastrini \\ Investigador y docente (UNQ /UBA / Conicet)
}

\section{Referencia de este artículo}

Mastrini, Guillermo (2021). Enrique Bustamante: un maestro en comunicación. En: adComunica. Revista Científica de Estrategias, Tendencias e Innovación en Comunicación, $\mathrm{n}^{0} 22$. Castellón de la Plana: Departamento de Ciencias de la Comunicación de la Universitat Jaume I, 381-398. DOI: http://dx.doi.org/10.6035/21740992.2021 .22 .22

Enrique Bustamante falleció el 20 de junio de 2021 en su Málaga natal. Pocas personas han influido tanto en el campo de las ciencias de la comunicación y, desde luego, era el principal investigador de la economía política de la comunicación en España. Sus trabajos sobre las transformaciones del sector audiovisual en las últimas décadas son referencia obligada para quienes estudian la comunicación contemporánea.

Su trabajo, primero como periodista y luego como académico, se destacó no sólo por la rigurosidad y excelencia de su producción, sino también por el permanente esfuerzo por articular su capacidad profesional con el contexto político y social. Enrique Bustamante se destacó por su valiosa contribución a un mejor conocimiento del funcionamiento de los sistemas de comunicación social, pero también por impulsar cambios y que mejoras que deriven de sus conocimientos científicos en un ecosistema de medios más democrático y plural.

Realizar un recorrido de la producción intelectual de Enrique Bustamante no resulta una tarea sencilla dada la vastedad y diversidad de la misma. Para intentar 
dar cuenta de la mejor manera posible su trayectoria hemos dividido este trabajo en tres partes. En primer lugar, analizaremos sus inicios en el periodismo y su actividad gremial y política, entendiendo que dicho período formativo constituye un antecedente fundamental para su producción académica posterior. En segundo lugar, se analizará la participación de Enrique Bustamante en proyectos académicos y políticos de intervención en la vida social y política, que trascienden las competencias tradicionales de los profesores que se encierran en sus aulas y teorías. Se destacan en este aspecto, la fundación y sostenimiento por décadas de la Revista Telos, la participación en la Comisión de Sabios para la Reforma de la Televisión española, y su activo rol protagónico en el grupo de pensamiento Teledetodos. Finalmente, se abordará con mayor detenimiento los aspectos principales de su producción intelectual, que por su riqueza no puede ser presentada de forma total, sino que se elegirán los aspectos que se estiman más destacados.

\section{Antes de la academia: ejercicio profesional y práctica política}

Enrique Bustamante nació en Málaga en 1949. Ya joven, en 1969, se traslada a Madrid, y aún durante el período franquista inició su formación profesional en la Escuela Oficial de Periodismo, ya que los estudios en comunicación social o ciencias de la información no habían sido institucionalizados en España aún. Los cuatro años de carrera de periodismo le permiten, no sólo familiarizarse con las prácticas profesionales de la época sino que también resultan una introducción a la vida política dentro de la proscripta izquierda española.

Una de sus primeras relaciones que lo ayudarían a entrar en el campo profesional fue la del hoy catedrático de la Universidad de Málaga, Bernardo Díaz Nosty con quien trabajó en la revista Criba. También desarrolló su labor periodística en Prensa del Estado, en carácter de prácticas profesionales, y colaboró establemente en la revista Triunfo, caracterizada como de oposición al régimen.

Simultáneamente continuó su formación como sociólogo en la Universidad Complutense de Madrid. En aquellos momentos, los campos de las ciencias sociales todavía se encontraban en fase de redefinición. Si bien ya se había producido la separación entre Ciencia Política y Sociología por un lado, y Económicas por el otro, la formación en los tres aspectos seguía siendo rigurosa. La articulación de estudios económicos, políticos y de sociología de la cultura, constituirán sin dudas uno de los principales aportes académicos de la obra de Enrique Bustamante.

Después, trabaja durante tres años en la revista Contrapunto, publicación de economía para ejecutivos que constituirá el lanzamiento como empresario de prensa de Jesús Polanco, futuro fundador y accionista dominante del diario El Pais durante años.

Luego de Contrapunto, participó de manera activa en la publicación Cuadernos para el Diálogo, donde trabajó junto a destacados periodistas como Joaquín Es- 
tefanía, Soledad Gallego Díaz y Vicente Verdú. Cuadernos para el Diálogo había surgido en octubre de 1963, de la mano de Joaquín Ruiz-Giménez, pero se publicaba como semanario desde 1974 y pervivió hasta finales del año 1978. Esta revista, con un origen democristiano, constituyó un importante espacio donde se aglutinó el pensamiento progresista en los últimos años del franquismo y los primeros de la transición, incluyendo socialistas y comunistas y sufrió por ello una permanente represión del régimen, con multas, expedientes y cierres temporales. En su editorial de libros, en 1974, Bustamante publicó «La cultura vasca, hoy (apuntes para un estudio)», con dibujos de Agustín Ibarrola, un informe sobre la cultura vasca, pionero tras la guerra civil, que constituye una de sus primeras aproximaciones a lo que luego sería uno de sus principales objetos de estudio, la cultura y su diversidad en España.

Mientras tanto, Bustamante desarrolló una activa vida política que lo llevó a militar en diversas agrupaciones de izquierda, principalmente el Partido Comunista y luego el PC Internacional, transformado en Partido del Trabajo, que fusionado con ORT en 1979, formaría el Partido de los Trabajadores, disuelto finalmente en 1980.

Complementaba su actividad política con una intensa participación en el gremio de prensa, destacándose su actuación en la Asociación de la Prensa de Madrid, en la Comisión de Defensa de la Profesión Periodística, donde fue elegido miembro de la Junta Directiva durante cuatro años y llevó a cabo una importante lucha por el estatuto profesional que derivaría en dos de las más importantes huelgas del sector (en defensa del secreto profesional y contra el atentado terrorista a la revista $E l$ Papus). También fue uno de los fundadores de la Unión de Periodistas de España y creó la agencia de noticias Argos Press con otros profesionales.

Las dificultades evidentes que creaba su participación política en el ejercicio del periodismo, hecho que lleva a su inclusión en las listas negras de varios medios, lo hacen pensar en un cambio de profesión.

Al igual que otro destacado investigador de la economía política de la comunicación como es Nicholas Garnham, Bustamante llegó a la academia luego de una intensa vida profesional y gremial. Si el británico había trabajado como productor audiovisual, el malagueño lo hizo luego de más de 12 años de ejercicio del periodismo. Como él mismo reconocía, su recorrido profesional tendrá importante injerencia en su trabajo académico, ya que sus análisis sobre el funcionamiento de los medios masivos de comunicación tienen como base su conocimiento interno de los mismos.

A fines de los años setenta, mientras se alejaba paulatinamente del ejercicio profesional del periodismo, Bustamante cursa sus estudios de doctorado en Sociología, que culminará en el año 80, con su tesis doctoral El poder de los medios de comunicación. Los amos de la información bajo la dirección de José Vidal Beneyto. En el año 1979 participó del mítico congreso sobre comunicación y cultura realizado en la ciudad de Burgos donde comienza a relacionarse con quienes tendrá un 
profundo intercambio a lo largo de su vida académica: Armand Mattelart, Ignacio Ramonet, Bernard Miege o Patrice Flichy. También se relacionó con investigadores de la comunicación de América Latina, con quienes mantendrá un fluido intercambio. El conjunto de estos autores constituirá las bases de sus referencias teóricas. Luego de aprobar su tesis doctoral, y a través de una invitación del profesor Antonio Lara, Bustamante comenzó a dar clases en el departamento de Comunicación Audiovisual de la Facultad de Ciencias de la Información que había sido creada en 1971. Originalmente dictó cursos de Teoría de la Imagen y de la Comunicación Audiovisual, pero ya desde entonces se preocupaba por el contexto sociopolítico y económico en el que operan los medios de comunicación y va insertando estos temas en sus asignaturas.

Durante la primera mitad de los 80 , y mientras formaliza el pasaje del periodismo a la academia, Bustamante entabló relaciones con colegas como el británico Philip Schlesinger, y sus connacionales Miquel de Moragas, Emili Prado y Ramón Zallo, con quienes darán un impulso en los años venideros a los estudios de comunicación en España y Europa. Durante el año 81, se trasladó varias semanas a París para estudiar las políticas culturales del partido socialista francés que había llegado al poder de la mano de François Mitterand. Ya en 1984, fundó junto a estos y otros colegas como Nicholas Garnham, Bernard Miége, Philip Schlesinger o Giusepe Richeri, el Eurocommunication Research Group, uno de los centros europeos de pensamiento comunicacional más importantes de la época, con numerosas publicaciones e importante influencia en la formación de jóvenes doctores europeos en el área, para los que van a organizar luego durante diez años un European Doctoral Workshop.

En 1985 propuso a la fundación Fundesco - Fundación para el Desarrollo de la Función Social de las Comunicaciones- de Telefónica la creación de una publicación sobre investigación en comunicación y forma parte del lanzamiento de la Revista Telos en calidad de director, pero esto ya es parte de otra historia.

\section{Entre la academia y la acción: intervención política y construcción de agenda progresista}

Antes de profundizar en el trabajo estrictamente académico de Enrique Bustamante, resulta conveniente analizar intervenciones de carácter más público como Telos, la comisión para la reforma de los medios de propiedad estatal, y la plataforma Teledetodos. Demás está decir que la participación de Bustamante en la vida política y social trascendió ampliamente estos tres ejemplos significativos de la forma en que el académico español considera el rol del intelectual pero se anotan también otras intervenciones como en la Universidad Internacional Menéndez Pelayo, en la fundación Alternativas, en Andalucía, Extremadura o Canarias. 
La revista Telos, si bien podría encuadrarse en el ámbito estrictamente académico, es analizada como una intervención pública debido a su incidencia no sólo en el campo científico sino también como generadora de debates sociales, especialmente en el ámbito iberoamericano, de la relación entre tecnología, medios y sociedad. La participación en la Comisión de Sabios sobre RTVE, es una muestra de la capacidad de articulación de Bustamante de los saberes académicos con la acción estatal y el diseño de políticas públicas. Y ante las propias limitaciones del ámbito de la política, y el fracaso de la reforma, Bustamante contribuyó a generar el grupo y portal Teledetodos, un espacio de intercambio y construcción de la sociedad civil donde continuó reclamando un espacio audiovisual democrático, además de colaborar con artículos en Le Monde Diplomatique en español, que dirige Ignacio Ramonet, desde 2003 a 2013.

\section{La Revista Telos}

En enero del año 1985, Enrique Bustamante fundó y asumió la dirección de la Revista Telos, una de las referencias centrales del campo de las ciencias de la comunicación en idioma español. Su aparición se dio casi en simultáneo con la aparición de las revistas Reseaux (en francés, con Patrice Flichy entre sus fundadores) y Media, Culture and Society (en inglés, con el liderazgo de Nicholas Garnham y Philip Schelesinger, entre otros). Si bien el propio Bustamante señaló que la coincidencia no es el resultado de un proyecto común, también destacaba que existían preocupaciones similares y compartidas. En el campo de las ciencias de la comunicación británico se había producido la ruptura entre los estudios culturales y la economía política de la comunicación. Las tres revistas, si bien no pueden identificarse exclusivamente con la economía política, van a dar numeroso espacio a los autores de esta corriente, que hasta entonces era marginal dentro del campo.

Por otra parte, Telos va a cumplir un rol fundamental en la articulación de académicos de Iberoamérica y autores de habla sajona y francesa. Sus páginas no sólo estimularon las traducciones al idioma de Cervantes de las principales líneas de investigación de la época, sino que al mismo tiempo permitieron que los académicos latinoamericanos fueran descubiertos por sus colegas europeos más curiosos, con numerosos autores colaboradores pero especialmente con tres dossiers dedicados al pensamiento latinoamericano. Facilitar ese punto de encuentro fue otro de los aportes de Enrique Bustamante.

En el primer número de Telos se destacó que la revista procuraba constituir un espacio de pensamiento que articulara alternativas concretas y soluciones claras desde la perspectiva crítica de la comunicación. Si bien el énfasis estaba puesto en la articulación entre tecnología y sociedad, se dejaba clara la necesidad de «contribuir al diseño de una política nacional de nuevas tecnologías de información que permita adecuar la técnica a las necesidades y demandas sociales de toda la 
población». La tecnología puesta al servicio de la sociedad y no en el sentido contrario, constituyó una premisa que Bustamante tuvo como faro orientador para su trabajo académico, y al mismo tiempo una guía orientadora de la lógica de la revista a lo largo de vida. En dicha editorial se destacaba además la

\begin{abstract}
«necesidad de contribuir a impulsar la investigación desde un punto de vista y una metodología transdisciplinar, capaz de superar los enfoques particulares para abarcar la complejidad de las nuevas tecnologías, sus consecuencias económicas globales, culturales, sociales, su impacto sobre las prácticas políticas y las relaciones internacionales».
\end{abstract}

Desde sus primeros años, los principales referentes de la economía política encontraron en las páginas de Telos un marco propicio para divulgar los avances de sus investigaciones en castellano. Se destaca la participación de Nicholas Garnham, Phillip Schlesinger, Bernard Miege, Armand Mattelart, Gaëtan Tremblay, Giuseppe Richeri y Ramón Zallo entre otros.

Paralela y periódicamente, Telos sostuvo una preocupación manifiesta por dar a conocer los estudios en comunicación en América Latina. Diversos dossiers que contaron con la participación de Jesús Martín Barbero, Néstor García Canclini, Héctor Schmucler, José Marques de Melo y Heriberto Muraro entre los más destacados, representan hoy un mapa de la evolución del pensamiento comunicacional en nuestra región. Pocos, por no decir ninguno, investigadores europeos sostuvieron con hechos la preocupación por divulgar el pensamiento latinoamericano en el campo comunicacional europeo como Enrique Bustamante. Esto es ratificado en la introducción al dossier de $2004\left(\text { Telos } n^{0} 61\right)^{1}$ donde Bustamante indica

«Entre los objetivos editoriales fijados por TELOS desde su primer número - hace más de veinte años ahora - figuraba con luz especial el de promover el mutuo conocimiento y la cooperación entre el mundo de la comunicación y la cultura latinoamericano, y sus analistas e investigadores, con el de España y la Península Ibérica y, por extensión, con el europeo».

Y constataba la evolución de los estudios de comunicación al indicar que

«tras una casual cadencia de siete-ocho años sobre las dos monografías anteriores, muchas cosas han cambiado sin embargo que exigen una re-orientación de su contexto y sus objetivos. De una parte, el pensamiento latinoamericano ha madurado seriamente en masa crítica y en calidad de la investigación. Por otro lado, felizmente, las desconexiones que se apreciaban en 1989 entre los investigadores de muchos países, a veces fronterizos, o las crisis de las incipientes instituciones de enlace de mitad de los años noventa parecen estarse solucionando en los últimos años».

El cierre de Fundesco marcó una primera crisis en la revista que dejó de salir entre 1997 y 2002. Pero ya con el aporte de la Fundación Telefónica retomó su tarea, manteniendo la preocupación por articular la investigación científica con el saber

1 Otros números de Telos que contaron con un dossier sobre Latinoamérica fueron el número 19 de 1989 y el 47 de 1996. 
hacer profesional y «colmar el foso entre los científicos sociales y los ingenieros y tecnólogos».

En 2008, con el impulso de Javier Nadal, se inició la tercera etapa de Telos, con la apertura a nuevos campos temáticos y una renovación y ampliación del Consejo Científico, con una notable expansión de sus lectores por Internet. Luego de 107 números editados bajo su conducción, Enrique Bustamante se retiró de la dirección de Telos en 2017, no sin haber dejado un aporte sustantivo al desarrollo del campo comunicacional, y un conjunto de reflexiones sobre las dimensiones económicas, sociales y políticas de las tecnologías en comunicación en español que no reconoce parangón en dicha lengua.

\section{La Comisión para la Reforma de RTVE}

Luego del desastre en el manejo de la información por parte de RTVE respecto a los atentados de Atocha en marzo de 2004 durante el período final del gobierno de José María Aznar, el presidente José Luis Rodríguez Zapatero decidió impulsar una reforma de los estatutos de la emisora. Para ello, resolvió convocar a un comité de expertos que debía elaborar una propuesta no vinculante. Enrique Bustamante fue uno de los cinco miembros, alcanzando un lugar en el que podía volcar toda su experiencia y su saber académico al diseño de políticas de comunicación del estado. El comité estuvo integrado además por el académico y catedrático de Filosofía Emilio Lledó (en carácter de presidente), y Victoria Camps, vicepresidenta del Consejo Audiovisual de Cataluña; el filósofo Fernando Savater, y Fernando González Urbaneja, presidente de la Asociación de la Prensa de Madrid. Bustamante para entonces había publicado varias investigaciones sobre la televisión, y era uno de los que poseía uno de los saberes más específicos dentro del comité, por lo que actuó como ponente de los sucesivos capítulos. La Comisión trabajó durante nueve meses y en febrero de 2005 presentó su informe que puede ser considerado un antecedente fundamental de la ley 17/2006 aprobada en julio de 2016, que reguló la radio y la televisión de titularidad estatal. Como reconoce el propio Bustamante:

\footnotetext{
«Hay que destacar que el Gobierno de Zapatero, que impulsó la reforma, contempló el $90 \%$ de las recomendaciones del informe. Lo que dejaron fuera, que parecían detalles, luego ha tenido muchísima repercusión. Como por ejemplo el tema de la elección de miembros del consejo de administración por mayoría simple, algo que con la llegada del Partido Popular al Gobierno acabaría por desnaturalizar la propia ley».
}

El informe se destacó por sostener un giro en la concepción del servicio público, promover la independencia informativa de los medios de propiedad estatal y estimular la modernización de la gestión de la corporación. El proyecto postulaba la necesidad de elegir a los directores por una mayoría calificada de los dos tercios del parlamento en lugar de la designación presidencial que había tenido hasta la fecha. Por otra parte, sostenía la necesidad de crear Consejos Informativos en 
las redacciones integrados por profesionales elegidos por votación directa, para controlar el comportamiento ético de los informativos. Estas medidas suponían avanzar, respectivamente, hacia la desgubernamentalización y hacia la mayor incidencia de los profesionales en el diseño de la línea informativa de los medios de la corporación pública.

Respecto a la definición de servicio público la prioridad estuvo puesta en los contenidos, con una preocupación por sostener la calidad de los contenidos. El Mandato de servicio público, debía ser revisable a largo plazo, con contratos-programa a plazo medio (cada tres años), que resultaban imprescindibles para justificar subvenciones presupuestarias, así como un procedimiento para verificar el cumplimiento de ese Mandato, conforme a la doctrina comunitaria europea.

Las designaciones de los directores de los medios estatales pasaban a requerir el acuerdo de los principales grupos parlamentarios, ya que se presupone que ningún grupo parlamentario alcanzaría con los dos tercios de los congresistas y acababa así con una tradición de más de un cuarto de siglo que había supuesto que el entonces director general fuera designado directamente por el Gobierno y para un periodo coincidente con la legislatura. A la vez, la ley 17/2006 estableció que los sindicatos mayoritarios en RTVE propusieran por primera vez a dos de los doce miembros del Consejo de Administración. Asimismo, el mandato de este órgano también se extendía de cuatro a seis años para garantizar que los relevos en los cargos de gestión de RTVE no corriesen paralelos a los cambios de Gobierno.

En términos generales, la propuesta del comité de sabios supuso un nuevo modelo de radiotelevisión pública «plenamente independiente» del Gobierno, con financiación mixta, prohibición de endeudamiento y con contenidos de servicio público de calidad.

Por su participación en el Informe, Enrique Bustamante recibió la concesión de la Gran Cruz de la Orden de Alfonso X el Sabio por parte del Gobierno de Zapatero.

Luego de aprobar la ley que reguló la televisión estatal en base al informe del comité de sabios, el gobierno implementó sus principales postulados. Sin embargo, a los pocos años realizó un giro en su política y produjo lo que Bustamante caracterizó como una contra-reforma en 2009. En lo que marca su carácter de pensador independiente, el haber participado de la comisión no le impidió ser uno de los mayores críticos de las medidas del gobierno que afectaron el financiamiento de los medios públicos. Tempranamente señaló:

\footnotetext{
"pero no cabe olvidar que es el propio Gobierno de Zapatero el que en 2009 ya empieza a hacer una contrarreforma. Por ejemplo, elimina la publicidad sin una memoria seria, crea corsés para RTVE con máximos de presupuesto. Y todo ello por la presión de las televisiones privadas que, para cuando se reforma la ley general del audiovisual en 2010, llega suprimir la publicidad en RTVE, sin prever una financiación alternativa sostenible».
} 


\section{Jornadas en Tenerife}

Entre 2007 y 2011, coordinó tres libros que recogían las contribuciones de destacados profesores: Bustamante mismo, Ramón Zallo, Rosa Franquet, Emili Prado, Luis A. Albornoz, José María Álvarez Monzoncillo, Patricia Corredor, Alejandro Perales, Javier López Villanueva, Gloria Gómez, Juan C. Calvi, Celeste Gay y Juan Carlos de Miguel- en unas jornadas organizadas a lo largo de un cuatrienio por el Cabido de Tenerife con acentos en las políticas, en el audiovisual y en Internet: Cultura y comunicación para el siglo XXI. Diagnóstico y política públicas, (2007), El audiovisual digital. Políticas y estrategias desde las Comunidades Autónomas (2009) y Las industrias culturales audiovisuales e internet. Experiencias, escenarios de futuro y potencialidades desde la periferia (2011), editados todos ellos por IDECO-Cabildo de Tenerife.

\section{Universidad Internacional Menéndez y Pelayo}

Dio carta de naturaleza a los estudios de comunicación ante la comunidad académica española al insertarlos, con seminarios y cursos específicos, en la Universidad Internacional Menéndez Pelayo (UIMP) de Cuenca y con posterioridad y en calidad de secretario general y vicerrector, en la sede central de Santander con su amigo y Rector Ernest Lluch, que sería asesinado por el ETA en el año 2000. Asimismo fue titular de la Cátedra Unesco de Comunicación Internacional en las universidades Stendhal de Grenoble y Lyon II.

\section{Teledetodos}

Luego de la frustración creada por el fracaso de las propuestas elaboradas por la Comisión de Sabios, Enrique Bustamante no se quedó satisfecho. Ya alejado de las tareas oficiales de la comisión, encaró un nuevo proyecto desde la sociedad civil para mantener vivo los aspectos fundamentales de la propuesta de contar con una radiodifusión pública de calidad. Junto a otros colegas, fundaron la plataforma Teledetodos (www.teledetodos.es) que se define como un grupo de estudio que agrupa a profesionales, académicos, investigadores y a todos aquellos ciudadanos o colectivos interesados en un auténtico servicio público de comunicación audiovisual y multimedia. Fundado en 2010, el colectivo está formado por más de 100 investigadores y profesionales de la información que funcionan como un think tank especializado en la reforma integral del servicio público.

Durante el año 2015, Teledetodos presentó el informe Un Nuevo Modelo para un Tiempo Nuevo. Diagnóstico del servicio público de Radio, Televisión y Servicios Interactivos. Propuestas para una Ciudadanía democrática, en el que se recoge una actualización, diez años después del Informe, de los principios aportados por Bustamante durante toda su vida académica. Esta vez enriquecido por los aportes de numerosos colegas y profesionales de los medios. 
Este trabajo cuenta con un diagnóstico que constata el deterioro del espacio público democrático en cuanto a pluralismo y diversidad, y especialmente la fuerte degradación de su servicio público en términos de audiencia, credibilidad y sostenibilidad financiera, con grave peligro de marginalidad en el próximo futuro.

La propuesta de Teledetodos parte de dos principios básicos, una apuesta decidida por la comunicación de servicio público y la convicción de que para tener una mirada crítica antes hay que estar dispuestos a edificar. Los promotores del informe coinciden en la necesidad de abrir un debate con la ciudadanía y con los grupos políticos que pueden determinar un modelo democrático, sostenible y de calidad que ya no puede esperar por más tiempo. Para ello proponen un gran pacto social entre la ciudadanía y el conjunto de partidos, que permita que los ciudadanos se apropien y controlen el servicio público, basado en primar la rentabilidad social sobre la económica, con total transparencia financiera.

El informe proponía un cambio cualitativo de la gobernanza, con auténticos Consejos Audiovisuales independientes y mediante la participación de la sociedad civil a través de Consejos Sociales y una plataforma interactiva de Ciudadanos por el Servicio Público.

En mayo de 2017, el parlamento español, con anuencia de toda la oposición mayoritaria, retomó las propuestas de la Comisión de Sabios para la elección del director y los consejeros de TVE, para eliminar el control que había ejercido sobre los medios de propiedad estatal el Partido Popular desde su llegada al gobierno, llegando a una proposición de ley aprobada casi unánimemente. Por supuesto que estos cambios no sólo se deben a la influencia de la sociedad civil, pero sin dudas la consecuente tarea de Enrique Bustamante al menos se vio parcialmente recompensada.

Antes de pasar a detallar sus trabajos como investigador, cabe destacar además que Bustamante contribuyó a crear la Fundación Alternativas y, dentro de ella, a dirigir una rama de investigación sobre la comunicación y la cultura, primero en el Laboratorio Alternativas, que encargó y editó más de medio centenar de investigaciones en este campo, y luego con la creación del Observatorio de Cultura y Comunicación, en el que Bustamante ha dirigido la edición de nada mensos que seis Informe(s) sobre el estado de la Cultura en España (2014, 2016, 2017, 2018, 2019 y 2020) que son referencia sobre evolución de la cultura en España tanto mediante análisis detallados como por el diagnóstico de opinión de sus agentes.

\section{La última reforma de RTVE}

Bustamante también formó parte del comité de expertos evaluador de los candidatos a formar parte del actual Consejo de administración de RTVE en el concurso público convocado en 2018. Tras dos años de trabajo y una maraña de impugna- 
ciones de la derecha española para hacer descarrilar el proceso de selección, $\underline{\text { se }}$ descartó la preselección por méritos de los aspirantes más idóneos y los grupos políticos recuperaron el mando para designar en Congreso y Senado por acuerdo partidario a los vocales del consejo y del presidente de la radio y la televisión estatal. La voz crítica de Bustamante no se hizo esperar: «Me parece una traición flagrante al espíritu de las leyes de 2006 y de 2017. Causará un mayor desprestigio del servicio público y agravará la caída de RTVE. Demuestra que el bipartidismo más pernicioso sigue vivo», declaró a El País.

\section{Extremadura y Andalucía}

Fue nombrado Doctor Honoris Causa por el Consejo de Gobierno de la Universidad de Extremadura en octubre de 2019, en agradecimiento por el apoyo a la implantación de estudios de comunicación en aquella universidad.

En comisión de servicios de la UCM, pasó a residir a su tierra natal, Málaga, entre 2017-2019, junto a su compañera y profesora Patricia Corredor, ejerciendo de Catedrático Investigador distinguido por encargo de las Universidades de Málaga y de Sevilla para realizar el Proyecto de Instituto Andaluz de Investigación en Comunicación y Cultura (INACOM), aprobado por los consejos de gobierno de ambas universidades en junio de 2019.

En los últimos meses había decidido volver a la Universidad Complutense de Madrid de la que era Catedrático Emérito.

\section{Una destacada producción académica}

Resulta imposible resumir en unas pocas páginas la voluminosa cantidad de textos e investigaciones académicas producidas por Enrique Bustamante a lo largo de su carrera. Pero al menos intentaremos reseñar cuáles han sido sus principales preocupaciones. En primer lugar, cabría destacar su trabajo para comprender el funcionamiento del mercado televisivo. En este sentido, Bustamante se ha preocupado por investigar tanto su funcionamiento básico, su perspectiva histórica, así como las transformaciones que ha implicado la digitalización, con especial atención a la actuación de la Unión Europea y de lo sucedido en Iberoamérica. En segundo lugar, se aprecia un trabajo sistemático por analizar las transformaciones de las industrias culturales en el contexto de la digitalización. Los volúmenes editados por Bustamante al respecto, constituyen una de las referencias ineludibles en lengua castellana. Si bien su trabajo ha estado orientado al estudio concreto de las industrias culturales con especial énfasis en la televisión, el debate teórico no le ha resultado ajeno. Por ejemplo, cuando ha intervenido con aportes significativos en el debate en torno al concepto de industrias creativas. Finalmente, no puede 
dejar de reseñarse su preocupación constante por el estado de la cultura en España, y sus más recientes trabajos sobre la cooperación cultural en Iberoamérica.

De esta forma, puede señalarse que las obras más importantes fueron: con Ramón Zallo (coordinadores) Las industrias culturales en España: grupos multimedia y transnacionales (1988), La televisión económica (1999); Historia de la radio y la televisión en España. Una asignatura pendiente de la democracia (Gedisa, 2013); Hacia un nuevo sistema mundial de comunicación. Las industrias culturales en la era digital (coord.), (2003); Comunicación y cultura en la era digital. Industrias, mercados y diversidad en España (coord.), (2004); e Industrias creativas. Amenazas sobre la cultura digital (2011).

«Las industrias culturales en España: grupos multimedia y transnacionales» fue la primera investigación en España que analizaba los procesos de concentración en cada una de las industrias cultuales con una sistemática común — prensa, radio, televisión, libro, cine, disco y publicidad- y un diagnóstico y para la que contó también con José Carrón, Rosa Franquet, Inmaculada Guiu, Ramiro Gómez Bermúdez de Castro y Daniel E. Jones.

Con La televisión económica Enrique Bustamante produjo un texto fundamental para la comprensión del funcionamiento del sistema televisivo a nivel mundial. Como señala el autor, la perspectiva económica había estado ausente en los debates sobre televisión. El libro constituye un estado del arte de la teoría sobre el mercado televisivo en su etapa analógica que aún conserva vigencia. El propio Bustamante se encargará de actualizar en investigaciones posteriores los desafíos que plantea la llegada de la televisión digital. En La televisión económica, Bustamante adelantaba dos cuestiones fundamentales para la economía política de este mercado. La primera es que sin considerar los aspectos económicos toda regulación pública estaba condenada al fracaso. La segunda es que se ha extendido la ilusión de que televisión comercial es gratis. En el libro, queda claro como la publicidad implica el pago de los usuarios implica un sostenimiento económico indirecto por parte de los usuarios. Tempranamente su investigación advertía sobre los nuevos modelos económicos que implicaba la irrupción con fuerza de la televisión de pago.

El libro destaca además el carácter de industria cultural líder de la televisión y el rol central en los nuevos escenarios que se estaban gestando entonces:

«la televisión en suma, como no podía ser menos en su papel de abanderada, viene a plantear ya en términos concretos y experimentales los grandes interrogantes de las nuevas redes de información. Al pensar y diseñar su futuro estamos también determinando en buena medida el de la llamada era de la información».

Si la televisión constituyó (y constituye) un oligopolio natural, sus transformaciones incluían la desregulación, la globalización, la integración en grupos más grandes, la convergencia y la digitalización. Todos estos temas que hoy son corrientes fueron tempranamente advertidos y estudiados por Bustamante en esta obra de referencia. 
Para los dos volúmenes sobre la transformación de las industrias culturales en el entorno digital, Bustamante reunió a un destacado grupo de investigadores que analizaron en diversas ocasiones las mutaciones que generaba la digitalización en la Cultura. El trabajo en colectivos que aportan y suman ideas es otra de las características de Enrique Bustamante. En esta ocasión, bajo una matriz común y un desarrollo que se sostuvo por más de cinco años se escrutaron los cambios que tuvieron lugar en las industrias del libro, la música, el cine, la prensa, la radio, la televisión, los videojuegos, así como las políticas públicas y la convergencia. Además de presentar la propuesta de investigación, Bustamante asumió los capítulos referidos a la televisión, que le permitieron actualizar su trabajo sobre la televisión económica.

Este trabajo tiene como objetivo analizar las transformaciones verificables que las industrias culturales muestran en las nuevas redes digitales y las tendencias observables que dibujarán presumiblemente los escenarios de futuro en este campo. Con acierto, los libros señalan el creciente peso de las industrias en la economía, al tiempo que mantienen su influencia cultural y política, en un contexto de concentración y globalización. La digitalización no resulta una innovación tecnológica neutra en este escenario.

La investigación se propone sostener un equilibrio entre los aspectos económicos, políticos y culturales, y sostiene que estas dimensiones permaneces entrelazadas aunque autónomas. Conceptualmente se sostiene dentro del concepto de industrias culturales, que encuentran como un concepto esclarecedor, en el que se sostiene la determinación mercantil e industrial de la mayor parte de la cultura contemporánea, justamente la de mayor trascendencia e influencia social.

Los trabajos de Bustamante y su equipo han devenido en un testimonio de las transformaciones fundamentales y las problemáticas transversales de las industrias culturales. En sus conclusiones se destaca una continuidad en los procesos de desregulación, concentración y globalización, hecho que los desmarca de la teoría del salto repentino. También se destaca que el impacto de la digitalización es diferencial según los diversos sectores que componen las industrias culturales. La investigación da cuenta de las acciones desarrolladas por los agentes analógicos para ocupar posiciones estratégicas en los soportes digitales, al tiempo que se produce un simultáneo proceso de desintermediación y el surgimiento de nuevos intermediarios. La disminución de los costes de creación y producción son acompañados por la flexibilización del trabajo creativo y técnico creativo.

Los trabajos cierran con una preocupación importante. El cambio de escenario, con sus incertidumbres no implican que las grandes preguntas respecto al futuro de la cultura sigan vigentes. Ante el optimismo tecnológico, los autores se preguntan si asistimos a un verdadero pluralismo o a un escenario de la mera diversidad que propone el mercado. Por ello las políticas culturales deben ser pensadas en este nuevo entorno, pero nunca abandonadas. 
Sin dudas, Historia de la radio y la televisión en España constituye la investigación a la que Bustamante dedicó mayor esfuerzo intelectual. Originalmente el trabajo partió de un encargo de la RAI, interesada por los servicios públicos en Europa, cuya editorial publicó un libro en italiano en dos volúmenes y más de 900 páginas, sintetizada por GEDISA en 2006 (30 aniversario de la creación de RTVE). Bustamante publicó una revisión y actualización de su pesquisa en castellano en 2013, llenando un vacío en la investigación, como destaca en sus primeras páginas, en las que realiza un llamado de atención sobre la escasa producción en la materia:

\footnotetext{
«Por un lado, sorprende que muchos historiadores que han prestado una notable atención a la producción intelectual y cultural no hayan tenido casi nunca en cuenta ni a la televisión ni a la radio, por contaminadas que pudieran estar por la cultura oficial. Por otro, la mayoría de los historiadores de la radio y la televisión han obviado el contexto histórico, político y económico de cada época, aunque, en ocasiones, datos y fechas sin conexión establecida con los procesos radiotelevisivos parecieran atender a ese requerimiento. Más aún, se ha olvidado con frecuencia el propio marco político y legal, social y cultural, de ideas y económico, que rodeaba a los medios de comunicación electrónicos en cada período».
}

La investigación de Bustamante repasa con exhaustividad la historia de los medios de propiedad estatal en España, con especial atención a la estrecha conexión entre política y radiotelevisión.

Del período franquista, Bustamante marca los límites que el servicio de la radiotelevisión española presentó para ser considerada servicio público: la falta de construcción y participación en una «esfera pública», la no consolidación de una «cultura nacional» al alcance de toda la nación, su organización como una rama del aparato del Estado y como su brazo propagandístico fundamental, en un marco de financiamiento publicitario que de acuerdo al autor era coherente con la dependencia gubernamental a ultranza.

Al final del régimen franquista y con la llegada de la transición democrática (19751982), Bustamante observa el nacimiento de un servicio público precario, que sin embargo mantiene características autoritarias y clientelares. Ni el partido gobernante ni la oposición lograron comprender la importancia de contar con un servicio público autónomo que fomentara el desarrollo de una esfera pública democrática.

Durante el período socialista (1982-1996), de acuerdo a Bustamante «la radiotelevisión pública quedó anclada en los resortes autoritarios del pasado, prácticamente sin cambios ni en la organización ni en la organización y la gestión ni en sus dependencias gubernamentales fuertes». En este período se consolida la comercialización de RTVE, que a partir de 1990 deberá con un sistema comercial privado que promoverá la crisis económica de las emisoras públicas y su endeudamiento. Como señala Bustamante, la incomprensión de los socialistas de las funciones e importancia de la radiodifusión pública en el período de Felipe González, se agrava por el contraste con otras políticas: «Y todo ello incluso en los períodos de bonanza 
económica y financiera de la década, y en medio de cierto esfuerzo por implantar el Estado de bienestar en otros campos».

Los gobiernos del Partido Popular encabezados por José María Aznar (1996-2004) reforzaron la dependencia gubernamental e incrementaron la crisis financiera de las emisoras estatales, que comenzaron a perder audiencia.

El período de Rodríguez Zapatero (2004-2011) como líder socialista, resulta clave por dos aspectos: porque constituyó un intento de transitar hacia un verdadero servicio público con un final poco feliz, y por la participación de Bustamante en la Comisión para la reforma de los mismos. Haber tenido lugar en dicho equipo no le impide a Bustamante realizar un análisis crítico de lo actuado en el período. Si bien destaca la promoción de medios estatales más plurales en los inicios del gobierno, al mismo tiempo se indican la línea zigzagueante de las políticas, hasta llegar a una contra-reforma que dio aire a las líneas más liberales del partido socialista, dando lugar a lo que Bustamante observa como un notable extravío del rumbo inicial.

Del período de Rajoy, aunque se limita a sus primeros años, la caracterización no podría ser más lapidaria, en el que se destacan los cambios en las formas de elección de los directivos de RTVE hasta transformar a la corporación en un adalid del gobierno.

En suma, la historia de la radiotelevisión en España constituye una obra imprescindible para comprender las conexiones entre política y medios, situadas en diversos contextos históricos. Un aporte al conocimiento de España y de los medios de comunicación.

Un cuarto trabajo que merece un relevamiento particular es la edición del volumen Las industrias creativas publicado en 2011. En dicho trabajo, Bustamante se da el gusto de reunir a varios de sus mejores colegas del campo de la comunicación en Europa para intervenir y criticar las formas de conocimiento que proponen simplificaciones de las características económicas de la cultura. Junto a Nicholas Garnham, Ramón Zallo, Giuseppe Richeri, Gaëtan Tremblay, Philip Schlesinger, Philippe Bouquillion, Bernard Miège, y Pierre Moeglin construyen un estado del arte en la crítica a la proliferación del concepto de industrias creativas, señalando claramente los límites del concepto, su tecnodeterminismo, y el fomento de la restricción al acceso a la cultura mediante la profundización del régimen de derechos de autor.

Los autores sostienen la necesidad de mantener políticas culturales específicas y diferenciadas, donde prime el valor de la diversidad, frente a la mercantilización acrítica planteada por los defensores de la economía creativa. De esta forma, sostienen la importancia de seguir investigando sobre la articulación de intereses ideológicos, económicos y de poder que se fraguan en la producción cultural, bien diferentes de la situación de mercados tales como la gastronomía, el turismo o el diseño. 
Como se ha indicado, la obra de Bustamante es tan prolífica que sólo pueden reseñarse sus principales trabajos. Sin embargos, resulta importante al menos rescatar su permanente interés por comprender el escenario latinoamericano, algo poco frecuente en investigadores europeos o norteamericanos. Su acogida y apoyo durante años a decenas de investigadores latinoamericanos, varios de ellos doctores con su dirección, ha enriquecido también este diálogo.

En un trabajo publicado en 2008, Bustamante analizaba críticamente la situación del audiovisual en la región y señalaba la necesidad de plantear políticas integrales para el sector, que englobaran al cine y la televisión. Debido a los rasgos estructurales compartidos entre América Latina y la Península Ibérica, Bustamante reclamaba mayor cooperación y articulación en la política cultural. A partir de comprender las lógicas económicas de la cultura y al mismo tiempo destacar la importancia de su influencia sobre la sociedad actual, el investigador malagueño observó la riqueza del espacio cultural iberoamericano pero al mismo tiempo la escasa capacidad para promoverlo y divulgarlo que han presentado hasta ahora sus políticas públicas.

\section{Palabras finales}

Como buen investigador que se precie, Enrique Bustamante no permaneció ajeno a las condiciones de trabajo en la que se desenvuelve el mundo académico en la actualidad. En oportunidad de un homenaje a su labor de investigación, Bustamante aprovechó para realizar una significativa denuncia del empobrecimiento de la situación del trabajo intelectual, con una especial referencia a los investigadores jóvenes, encargados de continuar y renovar las ideas:

\footnotetext{
«estas circunstancias suponen una presión extrema sobre los jóvenes investigadores, liderada por las ANECAS y las propias universidades (por mor de los rankings globales), que en mi opinión está teniendo efectos perversos desde hace unos años sobre la investigación en nuestro campo. Especialmente, destaco una presión productivista que valora la cantidad de méritos, ignorando la originalidad y la calidad; pero ligado a este vicio, me gustaría citar la marginación de los objetos de estudio incómodos, no correctos políticamente y poco susceptibles de ser valorados o apoyados o patrocinados, lo que incluye la imaginación de conclusiones críticas; o la proliferación de "metainvestigadores”, a quienes denomino así por su afición a investigar lo que investigan otros (a clasificarles, valorarles en sus temas, métodos, o soportes, nunca sobre sus resultados) lo que parece conferir un especial poder en esta época. Se está generando así un doble circuito perverso, dos mundos sin casi conexión: los seniors publican libros, los jóvenes publican artículos en busca obsesiva de las "estrellas Michelin". Los jóvenes leen y citan pocos libros, los seniors no siguen las revistas».
}

Pero al mismo tiempo que relevaba las carencias y dificultades del campo, Bustamante no dejó de visualizar un futuro con oportunidades. Su perspectiva del campo era esperanzadora y desafiante, crítica y alejada del confort que brinda el mercado: 
«En estas tres décadas transcurridas hemos visto cómo la Comunicación ha pasado de una concepción restrictiva, -el periodismo, los mass media-, a situarse en el centro del funcionamiento y del desarrollo de las sociedades contemporáneas. Comunicación sin pretensiones imperialistas, pero conformada cada vez más como encrucijada de caminos disciplinares múltiples, que se enriquece cada día con las aportaciones realizadas desde las perspectivas diversas de las Ciencias Sociales».

En este sentido el profesor Luis Albornoz remarcó que Bustamante ha sido reconocido por su análisis crítico de los fenómenos de la cultura y la comunicación, y por su lúcida preocupación por las problemáticas emergentes en estos ámbitos:

\footnotetext{
«Un análisis de la evolución de las tecnologías y de su enorme trascendencia económica y social para el presente de la sociedad alejado de una perspectiva tecnológico-determinista y de los discursos promocionales, siempre interesados, de las últimas aplicaciones de las llamadas “nuevas” tecnologías de la información y la comunicación».
}

Enrique Bustamante, reunió el compromiso y la vocación del intelectual que construyó un espacio para el pensamiento crítico en un marco de libertad y diversidad. Al fin al cabo es lo que el eligió hace ya tiempo para sí mismo y para quienes han trabajado en sus equipos. El placer de pensar independientemente, la libertad del espíritu crítico y el trabajo en equipos transversales, hayan sido posiblemente sus mayores enseñanzas y sus mayores placeres:

«La universidad pública es la última trinchera del pensamiento independiente en nuestro país. Crítico o no crítico, pero independiente, autónomo, no subordinado a intereses políticos o económicos. Desgraciadamente no sé cuánto tiempo durará así. Llevo ya 32 años en la universidad y me da satisfacciones impresionantes. La simple conciencia de mi independencia por encima de cualquier interés me produce una satisfacción maravillosa» (Heredia, 2013: 59).

\section{Publicaciones más relevantes}

Bustamante, Enrique (1982). Los amos de la información en España. Madrid: Akal. - (ed.) (1988). La televisión en España, mañana. Madrid: Siglo XXI.

- (1999). La Televisión Económica. Barcelona: Gedisa.

- (Coord.) (2002). Comunicación y Cultura en la Era Digital. Industrias, mercados y diversidad en España. Barcelona: Gedisa.

- (Coord.) (2003). Hacia un nuevo sistema mundial de comunicación. Las industrias culturales en la era digital. Barcelona: Gedisa.

- (2006). Radio y Televisión en España. Historia de una asignatura pendiente de la democracia. Barcelona: Gedisa.

- (Ed.) (2008). La Cooperación Cultura Comunicación en Iberoamérica. Madrid: AECID.

- (Coord.) (2011). Las Industrias Creativas. Barcelona: Gedisa. 
- (Coord.) (2011). Informe sobre la Cultura española y su proyección global. , Madrid: Fundación Alternativas:.

- (2013). Historia de la Radio y la Televisión en España. Barcelona: Gedisa.

Bustamante, Enrique; Francés, Miquel y Orozco Gómez (Coords.) (2021). La comunicación audiovisual en tiempos de pandemia. Barcelona: Gedisa.

Bustamante, Enrique y Álvarez Monzoncillo, José María (Eds.). (1999). Presente y futuro de la televisión digital. Madrid: Edipo.

Bustamante, Enrique y Zallo, Ramón (Coords.). (1986). Las industrias culturales en España. Madrid: Akal.

Villafañe Gallego, Justo; Bustamante, Enrique y Prado, Emili. (1988). Fabricar noticias. Barcelona: Mitre.

\section{Referencias}

Heredia, Daniel (2013). Entrevista a Enrique Bustamante Ramírez. En: Periférica, $\mathrm{n}^{0} 14$. Cádiz, Universidad de Cádiz, 49-59. doi: http://dx.doi.org/10.25267/Periferica.2013.i14.07 